\title{
Propuesta de un sistema de comunicación inalámbrico para una red de sensores bajo el agua en tiempo real aplicado a un sistema ROV
}

\section{Proposal of a Study and implementation of a wireless communication system for a network of underwater sensors in real time applied to a ROV system}

\author{
REYES-DE COSS, José Cuauhtémoc†*, RODRÍGUEZ-BLANCO, Marco Antonio y SÁNCHEZ- \\ LARA, Rafael
}

Universidad Autónoma del Carmen, Facultad de Ingeniería y Tecnología.

ID $1^{\text {er }}$ Autor: José Cuauhtémoc, Reyes-De Coss /ORC ID: 0000-0002-3419-905X, Researcher ID Thomson: AAP-30782020, CVU CONACYT ID: 920250

ID $1^{\text {er }}$ Coautor: Marco-Antonio, Rodriguez-Blanco / ORC ID: 0000-0003-3641-6895, Researcher ID Thomson: U-64762017, CVU CONACYT ID: 51908

ID $2^{\text {do }}$ Coautor: Rafael, Sanchez-Lara / ORC ID: 0000-0001-6587-1972, Researcher ID Thomson: AAP-3094-2020 CVU CONACYT ID: 88144

DOI: $10.35429 / J T E N .2020 .14 .4 .31 .39$

Recibido 03 de Junio, 2020; Aceptado 30 de Octubre, 2020

\section{Resumen}

En este trabajo se presenta una propuesta de un enfoque novedoso en comunicaciones de radio frecuencia aplicado a una red de sensores inalámbricas submarinas UWSN (Underwater Wireless Sensor Networks). La propuesta es manipular en un ambiente controlado y acotado, un vehículo operado remotamente ROV (Remote Operated Vehicle) sin cable umbilical mediante una red de sensores bajo el agua en base a comunicación por radio frecuencia, aprovechando las facultades de esta comunicación bajo el agua en conjunción con una red de sensores. Adicionalmente, se emplea redundancia dinámica en los transmisores hacia el ROV para incrementar el rango de alcance y evitar la pérdida de comunicación., además, se utiliza redundancia estática en el receptor ubicado en ROV para incrementar la confiabilidad en la comunicación. La parte de los sensores receptores del ROV se basa en una triple redundancia que facilita la toma de votos para poder obtener una tolerancia a fallas más optima. En la red de sensores se aplica redundancia dinámica en los transmisores para poder seguir manteniendo la comunicación con el ROV, en caso de falla se puede compensar la pérdida de comunicación con otro sensor de la red que tomara su función.

Red de sensores inalámbricas submarinas, Vehículo operado remotamente, Redundancia

\begin{abstract}
This work a novel approach in radio frequency communications applied to a underwater wireless sensors network UWSN is presented. The proposal is to manipulate in a bounded and controlled environment a Remote Operated Vehicle ROV without umbilical cable through a underwater sensors network based on radio frequency communication, taking advantage of the faculties of this underwater communication in conjunction with a sensor network, furter, a dynamic redundancy is used in the transmitters towards the ROV to increasing the range and avoiding communication loss, aditional a static redundancy is applied on the receiver to increase te reliability of comunication. The part of the ROV receiving sensors is based on a statisc redundancy of 3 sensors which will apply a vote taking in order to failure tolerantce, the sensor network will applied a dinamic redundacy in the transmitters in order to follow the communication with the ROV, in case of failure, the communication loss can be compensated with another sensor of the network that will take its role.
\end{abstract}

Underwater Wireless Sensor Networks, Remote Operated Vehicle, Redundancy

Citación: REYES-DE COSS, José Cuauhtémoc, RODRÍGUEZ-BLANCO, Marco Antonio y SÁNCHEZ-LARA, Rafael. Propuesta de un sistema de comunicación inalámbrico para una red de sensores bajo el agua en tiempo real aplicado a un sistema ROV. Revista de Ingeniería Tecnológica. 2020. 4-14: 31-39

\footnotetext{
$\dagger$ Investigador contribuyendo como primer autor.
} 


\section{Introducción}

Con el desarrollo de la microelectrónica, la electrónica, las TIC y la automática, se ha producido el avance de sistemas de adquisición inalámbricos (SAI) los que han impactado, de forma importante, en la salud, la monitorización de variables medioambientales, la agricultura de precisión (AP), la agropecuaria, la industria, los servicios, la domótica e inmótica. (Hurtado, 2020)

Usualmente las redes inalámbricas subacuáticas usan ondas acústicas como medio de transmisión actualmente (Partan, 2006), pero cabe decir que las posibilidades de los módems acústicos de obtener un mejor desempeño es muy remota y los enlaces ópticos submarinos no son muy prácticos en muchas aplicaciones (Murad, A. Sheikh, Asif Manzoor, Felemban, \& Qaisar, February 2015). Los requisitos modernos actualmente junto con las nuevas tecnologías de comunicaciones digitales nos obligan a reevaluar el papel de las señales electromagnéticas en aplicaciones submarinas.

En diferentes sectores, tanto en la industria petrolera, operaciones militares $\mathrm{y}$ ambientales exigen aplicaciones de enlace de datos confiables, sin conectores y de corto alcance por lo que las comunicaciones de radio frecuencia en el entorno submarino son una gran opción para los diferentes tipos de aplicaciones. (Che, Wells, Dickers, Kear, \& Gong, December 2010)

Con respecto a las comunicaciones submarinas existen tres tipos (Gkikopouli, Nikalakopoulos, \& Manesis, 2012), las comunicaciones acústicas que son actualmente las más utilizadas en diferentes aplicaciones submarinas, las comunicaciones ópticas que nos brindan una gran capacidad de transferencia de datos bajo el agua $\mathrm{y}$ por último las comunicaciones por radio frecuencia (ondas electromagnéticas) que carecen de algunas propiedades pero que nos ofrecen mejores beneficios en comparación con las otras.

Las comunicaciones acústicas submarinas son las más utilizadas actualmente, desde monitoreo costero, aplicaciones industriales o ya sea para uso militar ( $\mathrm{F}$. Akyildiz, Pompili, \& Melodia, 2005), este tipo de comunicación nos brinda una buena forma de comunicación bajo el agua (Liu, Zhou, \& Cui, July 2008).
Pero este tipo de comunicación conlleva consigo ciertos inconvenientes como el bajo rendimiento en aguas poco profundas, ancho de banda limitado, atenuaciones al transmitir entre el agua y el aire, adversamente afectado por turbidez, ruido ambiental, salinidad y gradientes de presión que son propios del ambiente pero sobre todo tiene un gran impacto perjudicial en la vida marina (M.G. Gussen, y otros, 2016). Las comunicaciones acústicas son la tecnología típica de capa física en redes submarinas. (Chitre Shiraz Shahabudeen \& Stojanovic, 2008)

Las ondas ópticas solo ofrecen un buen rendimiento en aguas muy claras y requieren una alineación estrecha de los nodos. El requisito de línea de visión cuando se utilizan comunicaciones ópticas ha impuesto una restricción, ya que la contaminación marina o el desarrollo de residuos propios del ambiente marino pueden obstruir la línea de visión impidiendo que la comunicación sea optima y confiable. (Farr, Bowen, Ware, \& Pontbriand, June 2010)

Por ultimo tenemos las comunicaciones por radio frecuencia (ondas electromagnéticas), las cuales presentan el inconveniente que son de corto alcance, pero presentan una serie de beneficios que las otras comunicaciones no ofrecen (Garcia, Sendra, Atenas, \& Lloret, May 2014), este tipo de comunicación no se ve afectada por la turbidez del entorno, la salinidad y los gradientes de presión que normalmente son propios del ambiente marino, no la afectan como las otros tipos de comunicaciones, no es afectado por el ruido acústico, y nos presenta un ancho de banda de hasta $100 \mathrm{Mb} / \mathrm{s}$ a corta distancia, lo cual para ciertos tipos de aplicaciones se puede llegar a aprovechar fácilmente y desarrollar aplicaciones en base a este tipo de características, este tipo de comunicación no afecta la vida marina por lo que es explotable a la hora de poder trabajar con ella debajo del mar sin temor a causar inconvenientes en la zona donde se aplica. (Che, Wells, Dickers, Kear, \& Gong, December 2010) Las comunicaciones por radio frecuencia (EM) en conjunto con la red de sensores aplicado a un sistema ROV haría posible la manipulación de una manera segura y eficaz, repasando todas las características que este tipo de comunicación nos ofrece, podemos observar que para aprovechar de mejor manera esta comunicación es ideal utilizar cortas distancias de transmisión, y en conjunto con la red de sensores ayudaría a optimizar.

REYES-DE COSS, José Cuauhtémoc, RODRÍGUEZ-BLANCO, Marco Antonio y SÁNCHEZ-LARA, Rafael. Propuesta de un sistema de comunicación inalámbrico para una red de sensores bajo el agua en tiempo real aplicado a un sistema ROV. Revista de Ingeniería Tecnológica. 2020 
(Sendra, Lloret, Jimenez, \& J.P.C. Rodrigues, 2016). Una red inalámbrica de sensores consiste en una gran cantidad de pequeños dispositivos, autónomos, distribuidos físicamente, llamados nodos de sensores, instalados alrededor de un fenómeno para ser monitoreado, con la capacidad de almacenar y comunicar datos en una red en forma inalámbrica. (GUADALUPE, 2019))

La mayoría de los robots submarinos no tripulados comerciales están atados con cables y operados a distancia (YUH, Design and Control of Autonomous Underwater Robots: A Survey, 2000), es decir que mucha de la tecnología actualmente utilizada en los ambientes submarinos utilizan un cable umbilical el cual provee la conexión entre el ROV y el operador (YUH \& WEST, Underwater robotics, 2011), los vehículos robóticos submarinos operados a distancia sin alambres pueden ser una solución en ambientes confinados bajo el agua en donde los cables umbilicales pueden ser elementos de obstrucción en la navegación y la operabilidad seguiría siendo remota. El problema principal en esta solución es la poca confiabilidad de la comunicación debido a la distancia y a los diversos factores involucrados bajo el agua (A. Moreno, y otros, 2014), por lo que la comunicación que mejor se adapta a esta problemática es la radio frecuencia (EM) en conjunto con la red de sensores.

La propuesta de este trabajo es adaptar un ROV(OpenROV), el cual cuenta con una estructura pequeña diseñada para hacer cómodo su transporte, cuenta con 3 motores thrusters (motores bruhsless), baterías de alimentación, cable umbilical y con un cilindro cerrado herméticamente el cual contiene una tarjeta tipo beagleBone que se encarga de procesar los diferentes comandos que se le pueden aplicar junto con todos los diferentes dispositivos con los que cuenta el OpenROV.

Para adaptar el OpenROV a un tipo de comunicación por radio frecuencia es necesario abrir el cilindro herméticamente cerrado y poder manipular la tarjeta interna donde se realiza el proceso de manipulación, para abordar este problema se adaptara este sistema alámbrico (cable umbilical) a inalámbrico (radio frecuencia), es diseñando la comunicación por radio frecuencia en otro cilindro herméticamente cerrado el cual podremos configurar de la manera más optima en conjunto con la red de sensores subacuáticos.

\section{Elementos propuestos}

$\mathrm{Al}$ comprender las diferencias entre cada tipo de comunicación que se puede efectuar bajo el agua se puede llegar a analizar cuáles son las ventajas y las desventajas de cada en (Che, Wells, Dickers, Kear, \& Gong, December 2010) nos explica en una tabla las diferencias entre cada tipo de comunicación. Las ventajas de la comunicación acústica es que es una tecnología probada con un alcance de hasta $20 \mathrm{~km}$, con una eficiencia energética, navegación de precisión, con bajo tamaño. No obstante, no transita atreves del agua al aire, pobres en aguas poco profundas y adversamente afectado por la aireación del agua, el ruido ambiental con propagación impredecible, su ancho de banda es limitado y con un impacto en la vida marina de manera que es detectable.

La comunicación óptica cuenta con ancho de banda ultra alto de hasta Gbps con un bajo costo, pero es susceptible a turbidez y partículas, y las propias del ambiente marino como son incrustaciones marinas en las caras de las lentes. La comunicación óptica necesita alineación ajustada a muy corto alcance, y como la comunicación acústica a esta también se le Dificulta transitar a través del agua al aire.

La comunicación por radio frecuencia (EM) transita el límite a través del agua al aire, también transita el límite agua al fondo marino y la señal puede atravesar el hielo. No se ve afectado por la profundidad del agua y no se ve afectado por turbidez/burbujas, tiene un rendimiento sin línea de visión, inmune al ruido acústico, es inmune a las incrustaciones marinas. La comunicación por radio frecuencia tiene velocidades de datos de hasta $100 \mathrm{Mbps}$ con una Capacidad ágil de frecuencia, esta no se ve afectado por la ruta múltiple y no se conocen efectos en animales marinos. Pero la comunicación es susceptible a interferencias electromagnéticas y tiene rango limitado a través del agua. Al adaptar nuestro sistema ROV a comunicaciones por radio frecuencias se podrá emplear un tipo de sensores de bajo costo (módulos RF $433 \mathrm{MHz}$ ) los cuales funcionan de forma unidireccional (un solo sentido).

Los módulos RF de 433Mhz son muy populares por su bajo costo y fácil uso. Vienen en pareja, emisor (FS1000A) y receptor (XYMK-5V), estos operan a una frecuencia de 433 $\mathrm{MHz}$ debido a que es una banda de uso libre (figura 1).

REYES-DE COSS, José Cuauhtémoc, RODRÍGUEZ-BLANCO, Marco Antonio y SÁNCHEZ-LARA, Rafael. Propuesta de un sistema de comunicación inalámbrico para una red de sensores bajo el agua en tiempo real aplicado a un sistema ROV. Revista de Ingeniería Tecnológica. 2020 


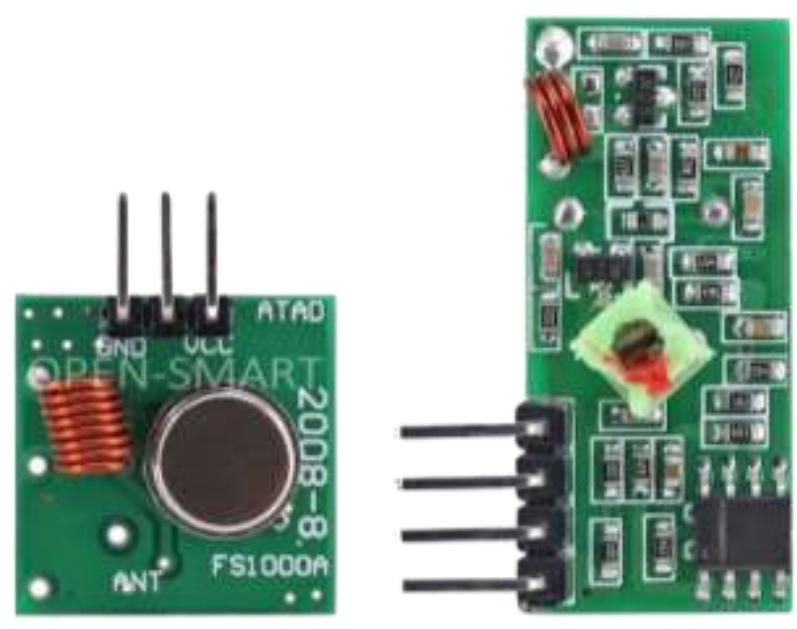

Figura 1 Modulo RF $433 \mathrm{Mhz}$ (emisor (Izquierda FS1000A) y receptor (Derecha XY-MK-5V)).

Fuente: Arduino communinity.

Estos módulos funcionan como transmisores en la red de sensores y como receptores en el ROV. Una vez establecido el tipo de sensores con el cual se trabaja en el interior del cilindro se pretende adaptar una tarjeta tipo Arduino UNO con las conexiones a los módulos RF $433 \mathrm{MHz}$ (figura 2).

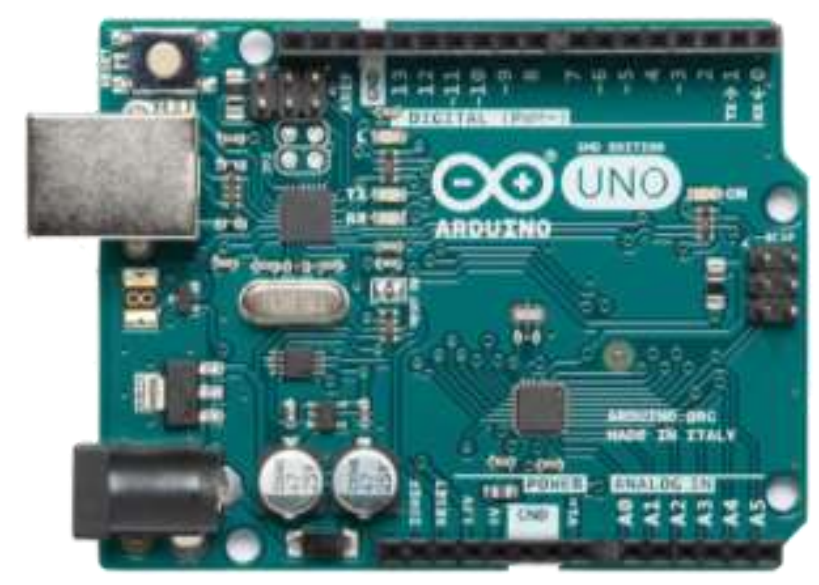

Figura 2 Arduino UNO

Fuente: Arduino communinity.

La tarjeta Arduino UNO se encargara de recibir las ordenes de los transmisores (emisor FS1000A) de la red de sensores, la cual enviara las señales que nos permitirán manipular el ROV de manera inalámbrica en un ambiente controlado, el Arduino UNO se encargará también de elegir cual señal obedecerá todo esto dependiendo de la cercanía a la que esté de los nodos de la red de sensores. De esta manera podremos aprovechar y expandir el distancia de movimiento del ROV gracias a la red de sensores. En la parte de transmisión conectaremos la Raspberry Pi con el Arduino para controlar sensores transmisores haciendo que estos dos se comuniquen a través del puerto serie.
Podemos combinar las ventajas que ofrece Raspberry Pi en cuanto sistema operativo como controlador maestro con las ventajas que ofrece Arduino para controlar diferentes sensores como esclavo, y todo a través del puerto serie. Los nodos de los sensores transmisores que estarán bajo el agua recibirán de manera alámbrica las ordenes mediante un Arduino conectado vía serial a una Rasberry PI 4 modelo B. (figura 3)

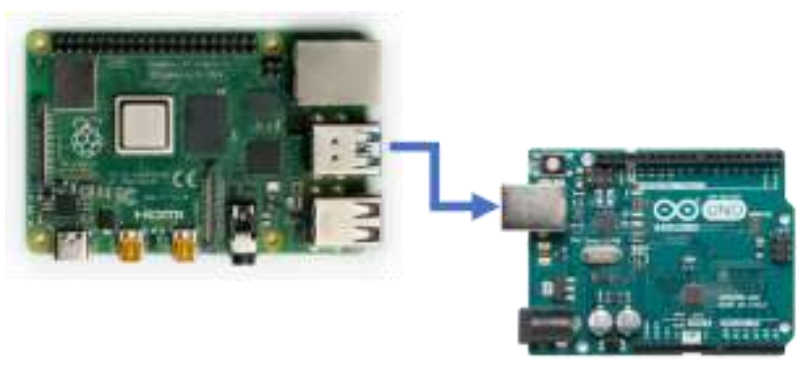

Figura 3 Arduino UNO conectado vía serial a Raspberry PI 4 model B

Fuente: Elaboración propia

La Raspberry Pi recibirá las ordenes de un mando con conexión vía Bluetooth que estaría previamente configurado y establecido hacia esta, aquí elegimos un control de Xbox ONE (figura 4) pero podría ser cualquier mando con Bluetooth que sea compatible con la Raspberry Pi, de modo que aprovecharemos la conexión Bluetooth de la Raspberry Pi.

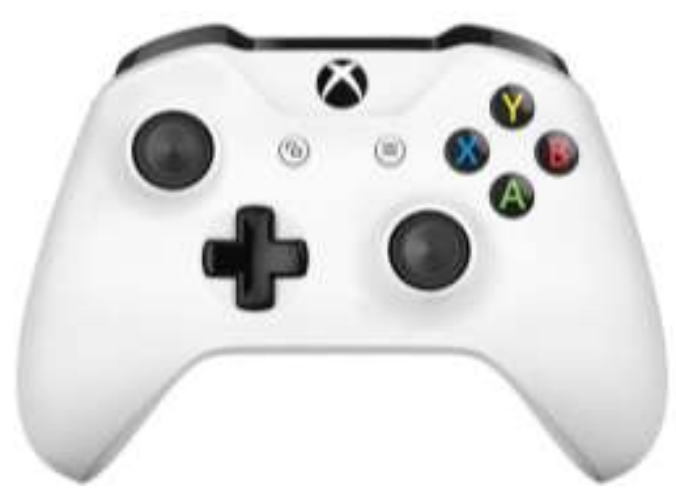

Figura 4 Mando Xbox ONE S

Fuente: Microsoft Xbox

La comunicación vía Raspberry Pi con Arduino UNO son previamente configuradas utilizando las librerías correspondientes para tener la correcta comunicación serial. Debido a que se propone establecer la comunicación en un nuevo cilindro, para poder manipular los motores thruster (motores bruhsless) se necesitan utilizar variadores de velocidad o ESC (Electronic Speed Controler) los cuales se encargan de definir la velocidad de giro del motor.

REYES-DE COSS, José Cuauhtémoc, RODRÍGUEZ-BLANCO, Marco Antonio y SÁNCHEZ-LARA, Rafael. Propuesta de un sistema de comunicación inalámbrico para una red de sensores bajo el agua en tiempo real aplicado a un sistema ROV. Revista de Ingeniería Tecnológica. 2020 
Debido a que la mayoría de los ESC giran en una sola dirección se optó por modificar el firmware de este para poder hacer posible el giro del motor en ambas direcciones, se utiliza un ESC de la marca HobbyKing de 20A UBEC (figura 6) que es uno de los más accesibles en el mercado.

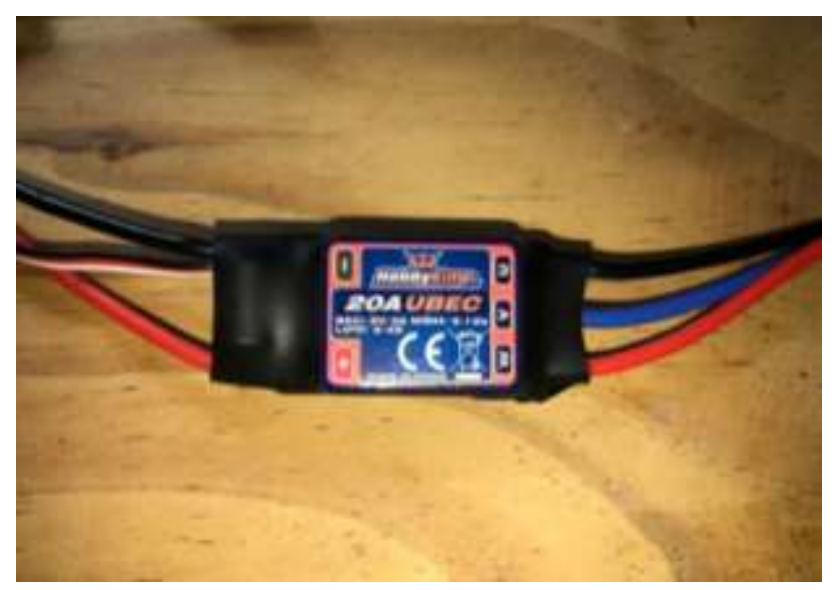

Figura 5 ESC 20A UBEC.

Fuente: HobbyKing.com

Para poder accesar al firmware y poder modificarlo utilizamos el programa KKmulticopter Flashtool en conjunto con una herramienta programadora Usbasp, accedamos al ESC con los pines que se encuentran en la parte posterior, este cuenta con 6 diferentes pines que son de forma ascendente: reset, +, -, sck, miso, mosi, que con los pines de la herramienta programadora deberemos mantener contacto en todo momento cuando se carga el nuevo firmware.

Se habilita la opción Reverse en el nuevo firmware y también se tiene la opción de colocar los parámetros con los cuales nuestro motor puede trabajar (Figura 6).

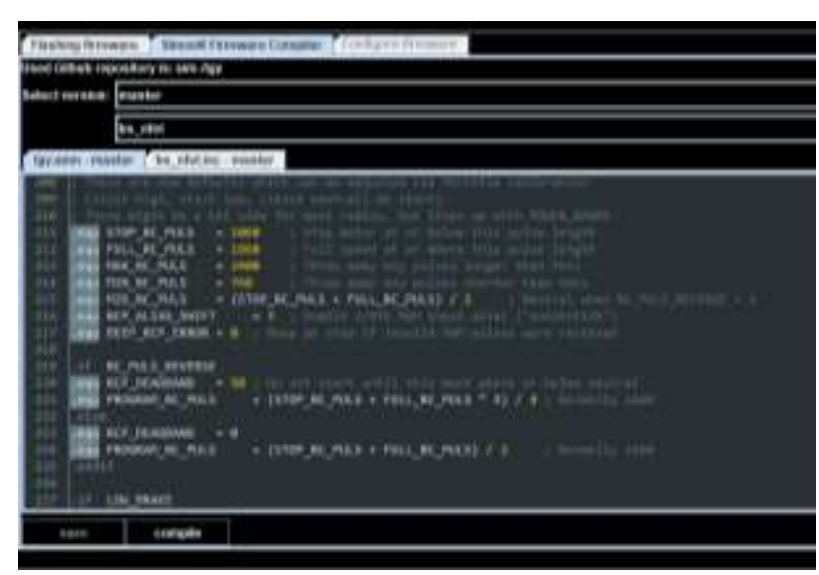

Figura 6 KKmulticopter Flashtool parametros.

Fuente: Elaboración propia

\section{Sistemas redundantes a fallas}

En este trabajo se implementará también los sistemas redundantes a fallas para mejorar la confiabilidad de la comunicación. Existen dos enfoques básicos para tolerar fallos en el marco de referencia de redundancia física: redundancia estática y redundancia dinámica. (Rodriguez, Claudio, Theilliol, \& Vela, 2007)

La redundancia estática utiliza " $\mathrm{n}$ " módulos activos con una misma señal de entrada y sus salidas se conectan a un evaluador de votos y se decide por mayoría si estas son correctas, dando como resultado el estado operacional de cada módulo (figura 7).

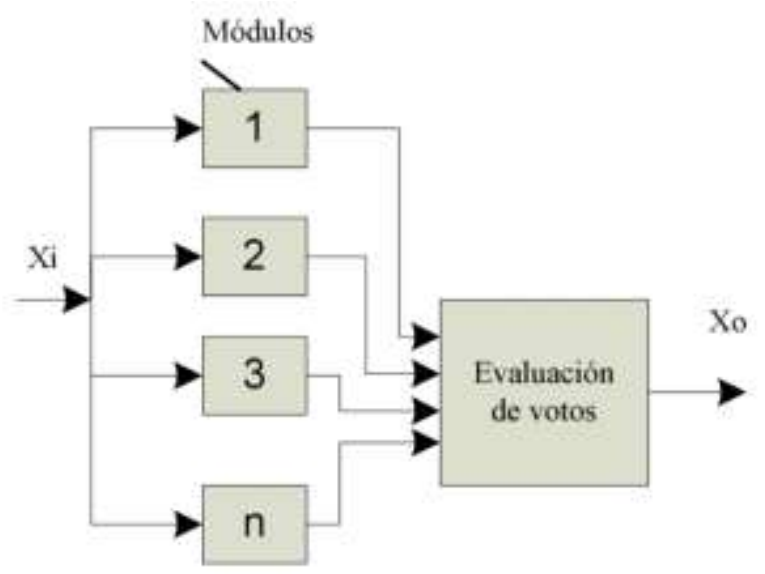

Figura 7 Esquema tolerante a fallo con redundancia estática

Fuente: Inversor para un Motor de Inducción Trifásico Tolerante a Fallos: Análisis y Diseño

La redundancia dinámica requiere de pocos módulos redundantes para tolerar un fallo, pero a consecuencia de mayor costo y procesamiento de información. Existen dos tipos de unidades de respaldo: hot-standby (a) y coldstandby (b).

En la unidad hot-standby el módulo de respaldo se encuentra trabajando continuamente y en cold-standby el módulo de respaldo solo se activa cuando ocurre el fallo, lo cual evita el envejecimiento del módulo de respaldo, pero requiere de dos interruptores adicionales para la etapa de aislamiento eléctrico y reemplazo del elemento dañado (figura 8). Sin embargo, para ambas unidades de respaldo, la utilización de la etapa de detección de fallos es esencial. 

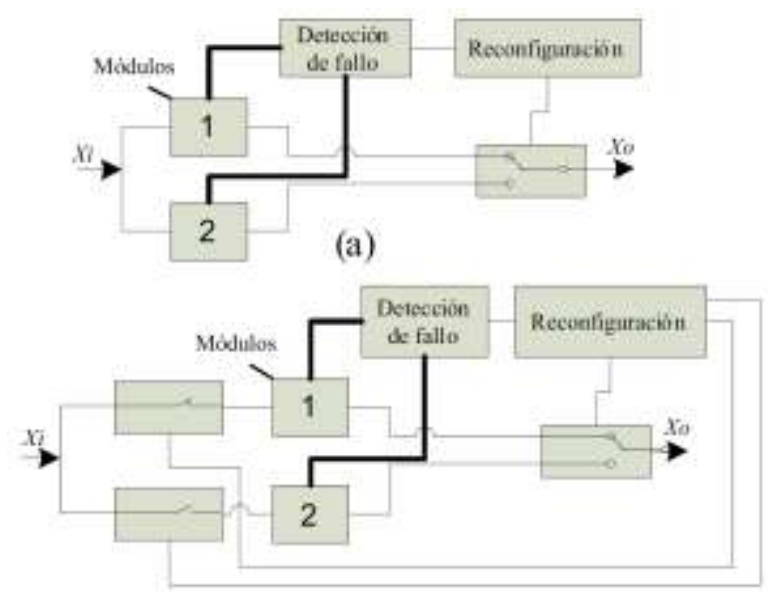

(b)

Figura 8 Esquema tolerante a fallo con redundancia dinámica y unidad de respaldo tipo hot-standby (a) y coldstandby (b).

Fuente: Inversor para un Motor de Inducción Trifásico Tolerante a Fallos: Análisis y Diseño

\section{Metodología}

La metodología por seguir será la que se muestra en el esquema.

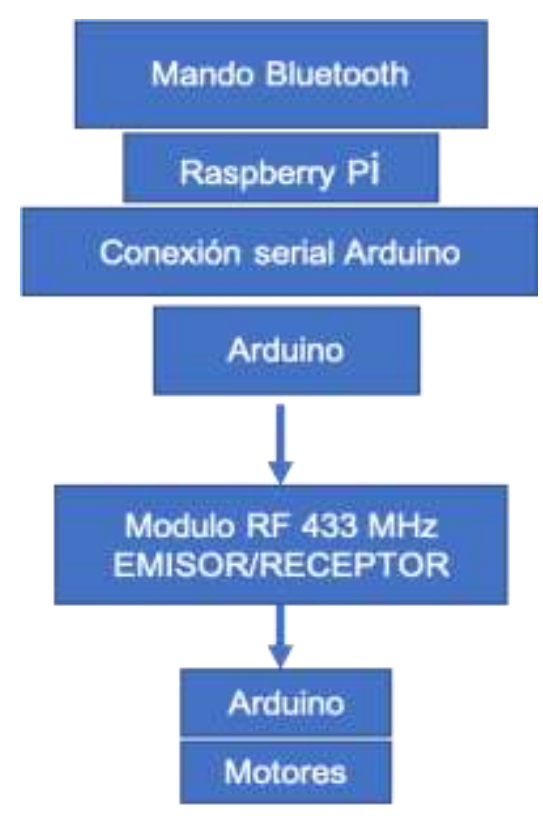

Figura 9 Metodología de comunicación Fuente: Elaboración Propia

Todo esto implementado un sistema redundante a fallas donde la redundancia estática estará en la parte receptora dentro del Arduino en el cilindro, donde reforzaremos así y prevendremos perdidas de señal en nuestro receptor, la redundancia dinámica estará en los nodos donde se aplicará redundancia tipo hotstandbye ya que los nodos estarán siempre transmitiendo en todo momento, aplicando una selección de señal.

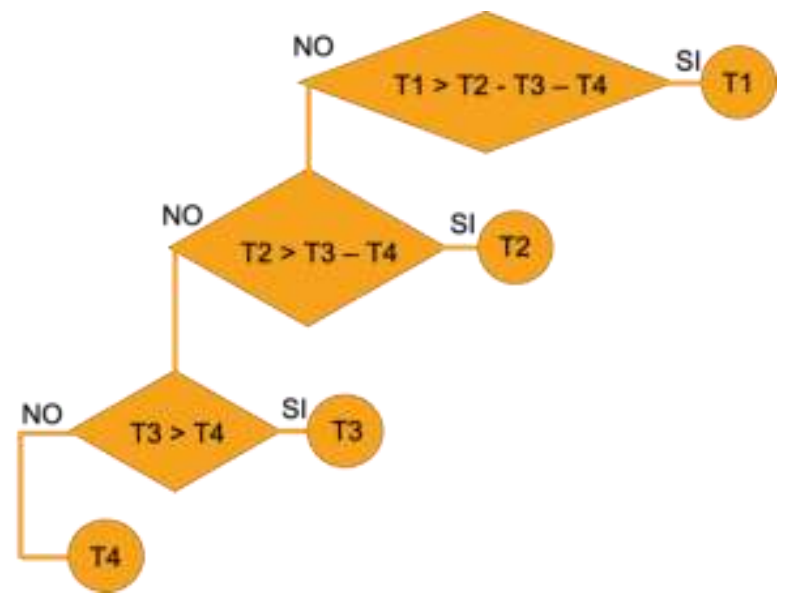

Figura 10 Selección de señal (donde T es el transmisor Fuente: Elaboración Propia

En la figura 10 se observa que dependiendo de la señal y de la posición del ROV este nos dirá a cuál de los emisores el receptor deberá recibir la señal, para esto se propone implementar un medidor de intensidad de potencia inalámbrica en la Rasberry Pi utilizando el enfoque RSSI (Received Signal Strength Indicator). (Jais, y otros, 2016). Otra manera de seleccionar la señal con mayor potencia es por desconexión de comunicación natural, para ello se propone un Detector de Pérdida de Comunicación DPC. Tal como se muestra en la figura siguiente.

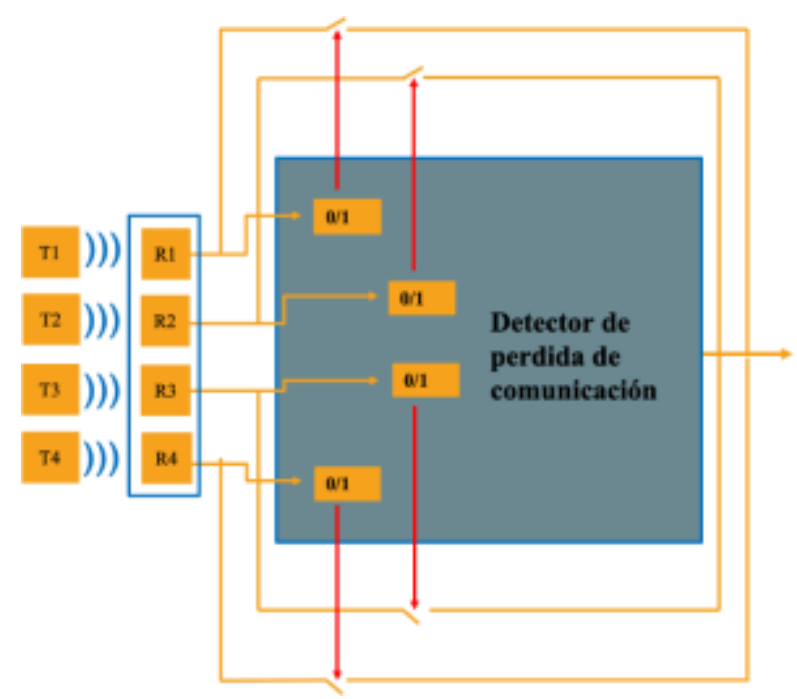

Figura 11 Detector de perdida de comunicación. Fuente: Elaboración Propia

En el esquema propuesto (figura 11), la señal más débil se desconectará por simple perdida de comunicación, esta será detectada por el bloque "Detector de perdida de comunicación" y conectara al transmisor inferior mediante elementos electromecánicos, si el transmisor seleccionado tiene una señal muy débil o la perdida de esta, el bloque de decidirá conmutar al siguiente transmisor inferior.

REYES-DE COSS, José Cuauhtémoc, RODRÍGUEZ-BLANCO, Marco Antonio y SÁNCHEZ-LARA, Rafael. Propuesta de un sistema de comunicación inalámbrico para una red de sensores bajo el agua en tiempo real aplicado a un sistema ROV. Revista de Ingeniería Tecnológica. 2020 
Esta propuesta es muy simple y evita la utilización de un esquema medidor de potencia, pero agrega un esquema medidor de perdida de transmisión lo cual es más fácil de implementar.

\section{Desarrollo de la señal}

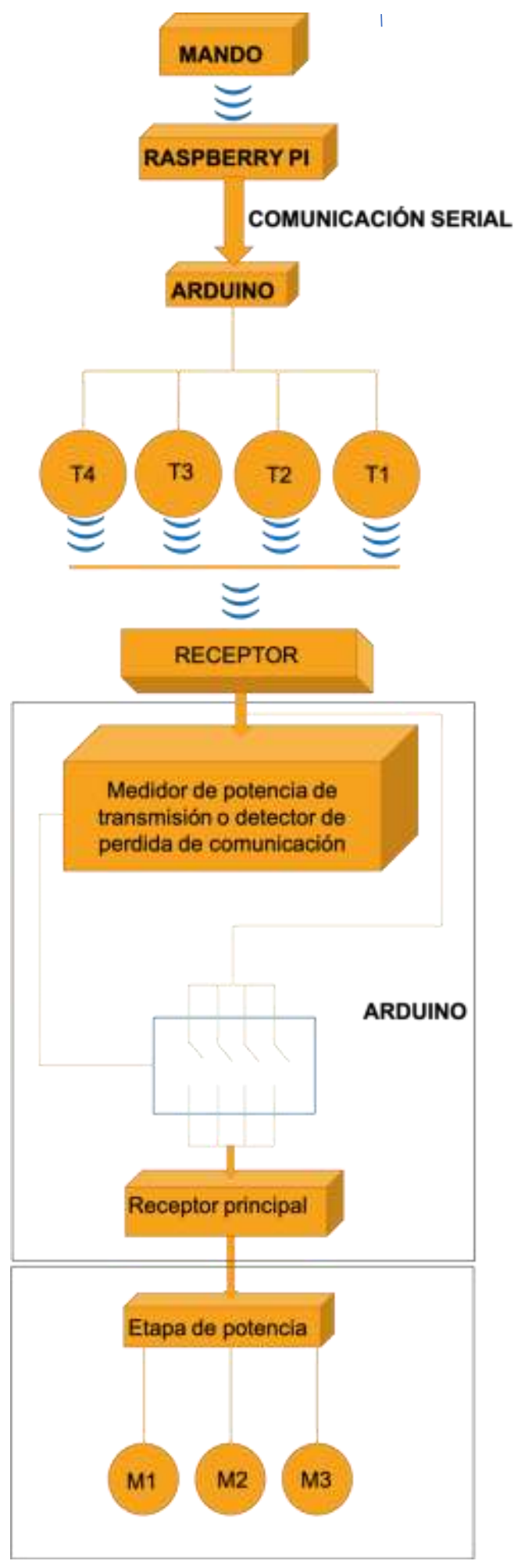

Figura 14 Esquema de transmisión. Fuente: Elaboración Propia
En el esquema propuesto observamos que la señal del mando conectado vía Bluetooth manda la acción a la Raspberry Pi, la Raspberry Pi envía la acción recibida vía serial al Arduino a los transmisores que son los nodos bajo el agua, los nodos transmitirán la señal en cada uno de ellos y el receptor recibirá la señal de uno de ellos dependiendo la posición que se encuentre, los receptores enviaran la señal al Arduino que procesara la acción y activara la etapa de potencia habilitando los motores.

\section{Pruebas experimentales}

Los módulos RF $433 \mathrm{MHz}$ han sido puestos a prueba dentro de unas boyas diseñadas y puestas bajo el agua para transmitir información, llegando a obtener una distancia de hasta $1.5 \mathrm{~m}$ de transmisión, con una separación del fondo de $15 \mathrm{~cm}$ con el suelo y $30 \mathrm{~cm}$ con la pared, además que se hundieron a una profundidad de hasta $1.35 \mathrm{~m}$, estas pruebas se realizaron con dos Arduino UNO enviando una serie de palabras.

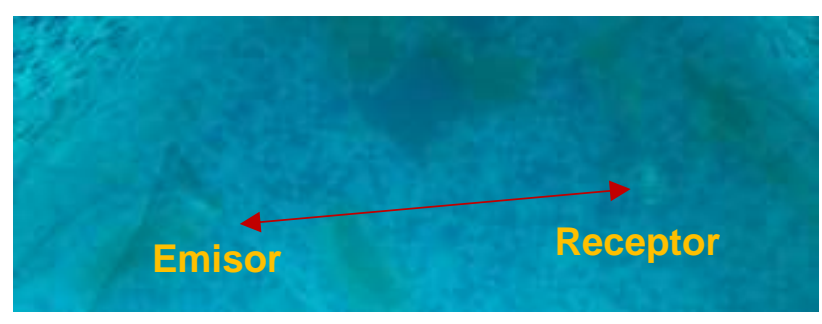

Figura 14 Comunicación entre dos Arduinos UNO con módulos RF433 Mhz

Fuente: Elaboración Propia

Con esta prueba podemos observar que la transmisión de datos bajo el agua usando radio frecuencia es viable.

Se adaptará la comunicación entre la Rasberry Pi y el Arduino UNO para poder controlar los motores del OpenROV modificado, y se propone implementar ya sea el indicador de intensidad de señal recibida (RSSI) o el detector de pérdida de comunicación DPC, los cuales son una medida estimada de lo bien que un dispositivo puede escuchar, detectar y recibir señales de cualquier punto de acceso. Lo bueno de RSSI o DPC es que le ayuda a determinar y saber si una señal es suficiente para establecer una conexión inalámbrica. 


\section{Agradecimiento}

Este trabajo fue financiado por la Universidad Autónoma del Carmen UNACAR y por el Consejo Nacional de Ciencia y Tecnología CONACYT de México.

\section{Conclusiones}

Como conclusiones se ha ondeado y puesto a prueba los módulos RF $433 \mathrm{MHz}$ bajo el agua, y se obtuvieron buenos resultados transmitiendo información a distancia de 1.5 metros entre un emisor y receptor. Una de las problemáticas que se enfrenta esta propuesta es la interferencia de señales lo cual podría ser mejorado encriptando las señales de comunicación. Otra problemática encontrada es la complejidad del algoritmo del indicador de intensidad de señal recibida RSSI en una plataforma comercial accesible económicamente como lo es la Raspberry Pi, lo cual podría ser mejorado utilizando un algoritmo simple de detección de perdida de comunicación DPC.

Con respecto a la redundancia dinámica, se recomienda utilizar elementos electromecánicos debido a que estos posen mayor aislamiento de entrada para evitar interferencia, aunque su conmutación es más lenta, lo cual no es indispensable para esta operación.

\section{Referencias}

A. Moreno, H., Saltarén, R., Puglisi, L., Carrera, I., Cárdenas, P., \& Álvarez, C. (2014). Robotica Submarina: Conceptos, Elementos, Modelado y Control. Revista Iberoamericana de Automática e Informática industrial , 1-17.

Che, X., Wells, I., Dickers, G., Kear, P., \& Gong, $X$. (December 2010). Re-Evaluation of RF Electromagnetic Communication in Underwater Sensor Networks. IEEE Communications Megazine, 143-151.

Chitre Shiraz Shahabudeen, M., \& Stojanovic, M. (2008). Underwater Acoustic Communications and Networking: Recent Advances and Future Challenges. Marine Technology Society Journal, 103-116.

Liu, L., Zhou, S., \& Cui, J.-H. (July 2008). Prospects and problems of wireless communication for underwater sensor networks. Wiley InterScience, 977-994.
F. Akyildiz, I., Pompili, D., \& Melodia, T. (2005). Underwater acoustic sensor networks: research challenges. Elsevier, 257-279.

Farr, N., Bowen, A., Ware, J., \& Pontbriand, C. (June 2010). An integrated, underwater optical /acoustic communication system. IEEE Xplore, 1-6.

Garcia, M., Sendra, S., Atenas, M., \& Lloret, J. (May 2014). Underwater Wireless Ad-hoc Networks: a Survey. ResearchGate, 1-35.

Gkikopouli, A., Nikalakopoulos, G., \& Manesis, S. (2012). A Survey on Underwater Wireless Sensor Networks and Applications. 20th Mediterranean Conference on Control \& Automation (MED) (págs. 1147-1154). Barcelona, Spain: IEEE.

GUADALUPE, T. L. (2019). SISTEMA DE MONITOREO DE FACTORES AMBIENTALES EXTERNOS EN UNIDADES EDUCATIVAS CÉNTRICAS DEL CANTON PILLARO BASADO EN TECNOLOGIA LORA. 1-29.

Hurtado, J. C. (2020). Sistemas de adquisición y accionamientos inálambricos utilizando hardware y dispositivs Android. (págs. 1-12). REVISTA CUBANA DE TRANSFORMACION DIGITGAL.

Jais, M. I., Sabapathy, T., Jusoh, M., Ehkan, P., Murukesan, L., Ismail, I., \& Ahmad, R. B. (2016). Received signal strength indication (RSSI) code assessment for wireless sensors network (WSN) deployed Raspberry-Pi. 2016 International Conference on Robotics, Automation and Sciences (ICORAS) (págs. 1-3). Ayer Keroh, Malaysia: IEEE.

M.G. Gussen, C., S.R. Diniz, P., L. R. Campos, M., A. Martins, W., M. Costa, F., \& N. Gois, J. (2016). A Survey of Underwater Wireless Communication Technologies. JOURNAL OF COMMUNICATION AND INFORMATION SYSTEM, 242-255.

Murad, M., A. Sheikh, A., Asif Manzoor, M., Felemban, E., \& Qaisar, S. (February 2015). A Survey on Current Underwater Acoustic Sensor Network Applications. International journal of computer theory and Engineering, 51-56.

REYES-DE COSS, José Cuauhtémoc, RODRÍGUEZ-BLANCO, Marco Antonio y SÁNCHEZ-LARA, Rafael. Propuesta de un sistema de comunicación inalámbrico para una red de sensores bajo el agua en tiempo real aplicado a un sistema ROV. Revista de Ingeniería Tecnológica. 2020 
Partan, J. (2006). A Survey Of Practical Issues in Underwater Networks. Computer Science Department Faculty Publication Series, 1-10.

Rodriguez, M., Claudio, A., Theilliol, D., \& Vela, L. (2007). Inversor para un Motor de Inducción Trifásico Tolerante a Fallos: Análisis y Diseño. Seminario Anual de Automática, Electrónica industrial

Instrumentación(SAAEI), 284-289.

Sendra, S., Lloret, J., Jimenez, J., \& J.P.C. Rodrigues, J. (2016). Underwater Communications for Video Surveillance System at $2.4 \mathrm{GHz}$. Sensors, 1-27.

Wells, I., Davies, A., Che, X., Kear, P., Dickers, G., Gong, X., \& Rhodes, M. (2009). Node Pattern Simulation of an Undersea Sensor Network using RF Electromagnetic Communications. IEEE, 1-4.

YUH, J. (2000). Design and Control of Autonomous Underwater Robots: A Survey. Kluwer Academic Publisher, 7-24.

YUH, J., \& WEST, M. (2011). Underwater robotics. Advanced Robotics, 609-639. 